\title{
Heavy quarkonium production in PQCD factorization
}

\section{Zhong-Bo Kang}

Theoretical Division, Los Alamos National Laboratory, Los Alamos, NM 87545, USA

E-mail: ZkangdLan L.gov

\section{Yan-Qing Ma*}

Physics Department, Brookhaven National Laboratory, Upton, NY 11973-5000, USA

E-mail: vamadion $1.90 \mathrm{v}$

\section{Jian-Wei Qiu}

Physics Department, Brookhaven National Laboratory, Upton, NY 11973-5000, USA

C.N. Yang Institute for Theoretical Physics and Department of Physics and Astronomy, Stony

Brook University, Stony Brook, NY 11794-3840, USA

E-mail: Gaiudbn1.9ov

\section{George Sterman}

C.N. Yang Institute for Theoretical Physics and Department of Physics and Astronomy, Stony

Brook University, Stony Brook, NY 11794-3840, USA

E-mail: stermandinsti.physics.sunysb.edu

\begin{abstract}
We present a factorization formalism for inclusive production of heavy quarkonia of large transverse momentum, $p_{T}$ at collider energies, including leading power (LP) and next-to-leading power (NLP) behavior in $p_{T}$. We demonstrate that both LP and NLP contributions can be factorized in terms of perturbatively calculable short-distance partonic coefficient functions and universal non-perturbative fragmentation functions, and derive the evolution equations that are implied by the factorization. We evaluate the first non-trivial order of evolution kernels for all relevant fragmentation functions, and discuss the role of NLP contributions.
\end{abstract}

XV International Conference on Hadron Spectroscopy-Hadron 2013

4-8 November 2013

Nara, Japan

${ }^{*}$ Speaker. 


\section{Introduction}

The most theoretically sound and phenomenologically successful model for heavy quarkonium production at present is the non-relativistic QCD (NRQCD) model [四]. However, recently complete next-to-leading order calculations within NRQCD method [ [ 2 , 及, 团] have difficulties to describe inclusive heavy quarkonia production at high energy colliders by using universal NRQCD longdistance matrix elements [ [5, 囵]. Motivated in part by these challenges to existing theories, new

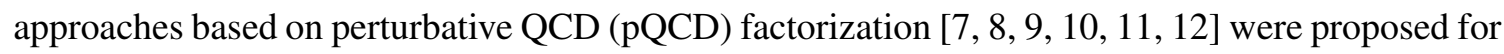
the systematic study of heavy quarkonium production at collider energies. A similar factorization approach based on soft-collinear effective theory was also recently proposed [[1], प44]. In the pQCD factorization approach, the cross section is first expanded by $1 / p_{T}^{2}$, and both the leading power (LP) and next-to-leading power (NLP) terms of the expansion are factorized systematically into infrared-safe short-distance hard collision convoluted with universal fragmentation functions (FFs) [ㄴ, ㅁ].

The predictive power of this new factorization formalism relies on the perturbative calculations of the short-distance functions and the evolution kernels, and our knowledge of the universal fragmentation functions at an input scale $\mu_{0}$. The first nontrivial order in the $\alpha_{s}$ expansion of evolution kernels and short-distance functions are given by Refs. [ए]] and [ए2], respectively. The input fragmentation functions are non-perturbative, and in principle, can be extracted from fitting experimental data. In Refs. [ㅍ, [6] , these fragmentation functions have been calculated by applying an NRQCD-inspired model.

\section{2. $\mathrm{pQCD}$ factorization}
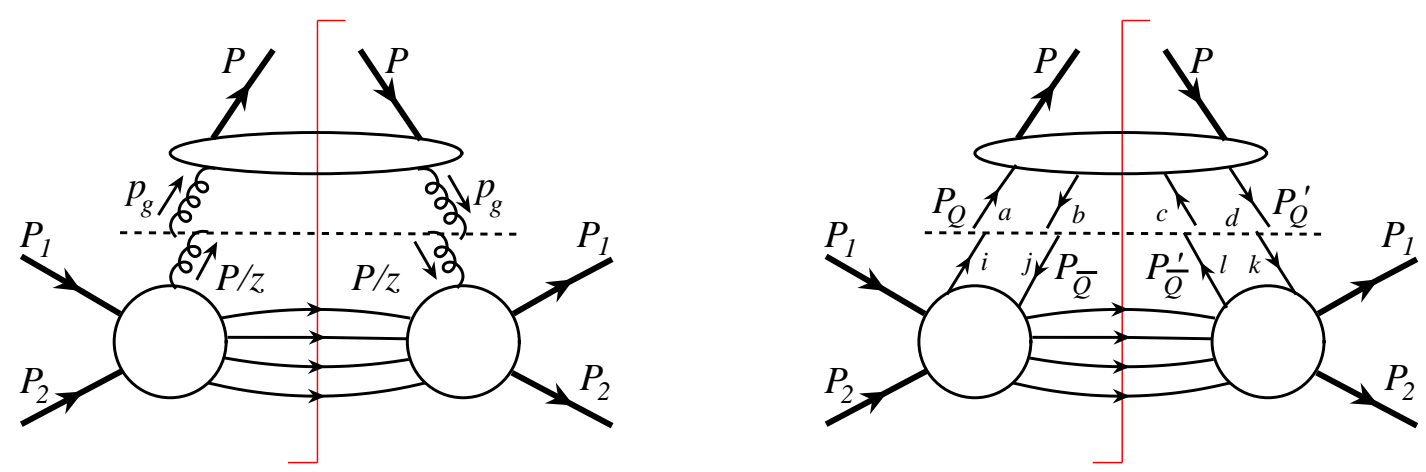

Figure 1: These generic Feynman diagrams represent alternative heavy quarkonium production processes, via the production of a single parton, here a gluon, (left) and a heavy quark pair (right) at short distance.

In terms of the $1 / p_{T}^{2}$ expansion, the first two power contributions to the cross section of heavy quarkonium production at high $p_{T}$ are presented in sample diagrams in Fig. W. With all contributions up to the first subleading power in $1 / p_{T}^{2}$, we have the corresponding factorization formula [ए]],

$$
E_{P} \frac{d \sigma_{A+B \rightarrow H+X}}{d^{3} P}(P) \approx \sum_{f} \int \frac{d z}{z^{2}} D_{f \rightarrow H}\left(z ; m_{Q}\right) E_{c} \frac{d \hat{\sigma}_{A+B \rightarrow f\left(p_{c}\right)+X}}{d^{3} p_{c}}\left(p_{c}=\frac{1}{z} p\right)
$$




$$
\begin{aligned}
& +\sum_{[Q \bar{Q}(\kappa)]} \int \frac{d z}{z^{2}} d u d v \mathscr{D}_{[Q \bar{Q}(\kappa)] \rightarrow H}\left(z, u, v ; m_{Q}\right) \\
& \quad \times E_{c} \frac{d \hat{\sigma}_{A+B \rightarrow[Q \bar{Q}(\kappa)]\left(p_{c}\right)+X}}{d^{3} p_{c}}\left(P_{Q}=\frac{u}{z} p, P_{\bar{Q}}=\frac{\bar{u}}{z} p, P_{Q}^{\prime}=\frac{v}{z} p, P_{\bar{Q}}^{\prime}=\frac{\bar{v}}{z} p\right) \\
& +\sum_{\left[f f^{\prime}\right] \neq[Q \bar{Q}(\kappa)]} \int \frac{d z}{z^{2}} d u d v \mathscr{D}_{\left[f f^{\prime}\right] \rightarrow H}\left(z, u, v ; m_{Q}\right) \\
& \quad \times E_{c} \frac{d \hat{\sigma}_{A+B \rightarrow\left[f f^{\prime}\right]\left(p_{c}\right)+X}}{d^{3} p_{c}}\left(P_{1}=\frac{u}{z} p, P_{2}=\frac{\bar{u}}{z} p, P_{3}=\frac{v}{z} p, P_{4}=\frac{\bar{v}}{z} p\right),
\end{aligned}
$$

where variables $z, u, v, \bar{u}=1-u$, and $\bar{v}=1-v$ are light-cone momentum fractions of fragmenting partons. Among the NLP contributions, the second term (i.e. $[Q \bar{Q}(\kappa)]$ fragmentation) should dominate because it should have a larger possibility to produce a heavy quarkonium since a heavy quark pair is already there. We thus ignore all other NLP contributions (the third term and twist-4 contributions in the first term) for now.

Each term in Eq. (2.]), either LP contribution or NLP contribution, comes from a specific pinch surface [ㅁ]], which has off-shell partons, on-shell collinear partons, as well as a "cloud" of soft partons interacting in all possible ways. In the NLP expansion, the most dangerous interaction that may ruin the factorization is soft lines attached the leading fragmenation jet to other jets at the pinch surface [W]. In this case, soft gluons can be factorized and canceled from all jets as long as soft momenta are not in the "Glauber region" [ए8]. For "Glauber" gluons, we can deform the $n$-component of soft gluons to be larger, which takes the corresponding jet lines to a larger off-shellness, and thus away from the pinch surface. We conclude that nonperturbative effects that violate the factorization are suppressed to next-to-next-to-leading power.

\section{Evolution kernels}

By demanding that a physical cross section for heavy quarkonium production should not depend on the choice of the factorization scale $\mu$, we obtain the following evolution equations for fragmentation functions up to NLP expansion [ए]],

$$
\begin{aligned}
\frac{\partial}{\partial \ln \mu^{2}} D_{f \rightarrow H}\left(z, \mu^{2} ; m_{Q}\right) & =\sum_{f^{\prime}} \int_{z}^{1} \frac{d z^{\prime}}{z^{\prime}} D_{f^{\prime} \rightarrow H}\left(z^{\prime}, \mu^{2} ; m_{Q}\right) \gamma_{f \rightarrow f^{\prime}}\left(z / z^{\prime}, \alpha_{s}\right) \\
+ & \frac{1}{\mu^{2}} \sum_{\left[Q \bar{Q}\left(\kappa^{\prime}\right)\right]} \int_{z}^{1} \frac{d z^{\prime}}{z^{\prime}} \int_{0}^{1} d u^{\prime} \int_{0}^{1} d v^{\prime} \mathscr{D}_{\left[Q \bar{Q}\left(\kappa^{\prime}\right)\right] \rightarrow H}\left(z^{\prime}, u^{\prime}, v^{\prime}, \mu^{2} ; m_{Q}\right) \\
& \times \gamma_{f \rightarrow\left[Q \bar{Q}\left(\kappa^{\prime}\right)\right]}\left(z / z^{\prime}, u^{\prime}, v^{\prime}, \alpha_{s}\right)
\end{aligned}
$$

and

$$
\begin{aligned}
\frac{\partial}{\partial \ln \mu^{2}} \mathscr{D}_{[Q \bar{Q}(\kappa)] \rightarrow H}\left(z, u, v, \mu^{2} ; m_{Q}\right)=\sum_{\left[Q \bar{Q}\left(\kappa^{\prime}\right)\right]} \int_{z}^{1} \frac{d z^{\prime}}{z^{\prime}} \int_{0}^{1} d u^{\prime} \int_{0}^{1} d v^{\prime} \\
\times \mathscr{D}_{\left[Q \bar{Q}\left(\kappa^{\prime}\right)\right] \rightarrow H}\left(z^{\prime}, u^{\prime}, v^{\prime}, \mu^{2} ; m_{Q}\right) \\
\times \Gamma_{[Q \bar{Q}(\kappa)] \rightarrow\left[Q \bar{Q}\left(\kappa^{\prime}\right)\right]}\left(z / z^{\prime}, u, v ; u^{\prime}, v^{\prime}, \alpha_{s}\right),
\end{aligned}
$$


where $\gamma_{f \rightarrow f^{\prime}}$ is the well-known leading power DGLAP evolution kernel, $\gamma_{f \rightarrow\left[Q \bar{Q}\left(\kappa^{\prime}\right)\right]}$ and $\Gamma_{[Q \bar{Q}(\kappa)] \rightarrow\left[Q \bar{Q}\left(\kappa^{\prime}\right)\right]}$ are new, both of which are calculated in Ref. [ए]] to the first perturbative order, while $\Gamma_{[Q \bar{Q}(\kappa)] \rightarrow\left[Q \bar{Q}\left(\kappa^{\prime}\right)\right]}$ was also calculated in an independent study [[1], [4]]. The effect of $\gamma_{f \rightarrow\left[Q \bar{Q}\left(\kappa^{\prime}\right)\right]}$ is to take into account contributions where a heavy quark pair is produced between time scales of $1 / p_{T}$ and $1 / m_{Q}$. As an example, we find

$$
\begin{aligned}
\Gamma_{[Q \bar{Q}(v 8)] \rightarrow[Q \bar{Q}(v 8)]}^{(1)}\left(z, u, v ; u^{\prime}, v^{\prime}\right) \\
=\left(\frac{\alpha_{s}}{2 \pi}\right)\left\{[ \frac { 1 } { 2 N _ { c } } ] \left\{\frac{1}{2} \frac{z}{(1-z)_{+}} S_{+} \Delta_{-}^{[8]}-\delta(z-1)\right.\right. \\
\quad \times\left[\delta\left(v-v^{\prime}\right)\left(\frac{\theta\left(\bar{u}^{\prime}-\bar{u}\right)}{\left(\bar{u}^{\prime}-\bar{u}\right)_{+}}\left(\frac{\bar{u}}{\bar{u}^{\prime}}\right)\left(\bar{u}^{\prime}+u\right)+\frac{\theta\left(u^{\prime}-u\right)}{\left(u^{\prime}-u\right)_{+}}\left(\frac{u}{u^{\prime}}\right)\left(u^{\prime}+\bar{u}\right)\right)\right. \\
+\delta\left(u-u^{\prime}\right)\left(\frac{\theta\left(\bar{v}^{\prime}-\bar{v}\right)}{\left(\bar{v}^{\prime}-\bar{v}\right)_{+}}\left(\frac{\bar{v}}{\bar{v}^{\prime}}\right)\left(\bar{v}^{\prime}+v\right)+\frac{\theta\left(v^{\prime}-v\right)}{\left(v^{\prime}-v\right)_{+}}\left(\frac{v}{v^{\prime}}\right)\left(v^{\prime}+\bar{v}\right)\right) \\
+\left[\frac{N_{c}}{2}\right] \delta(z-1) \delta\left(u-u^{\prime}\right) \delta\left(v-v^{\prime}\right)[3-\ln (u \bar{u} v \bar{v})] \\
\left.-\left[\frac{1}{2}\right] \delta(z-1)\left[\delta\left(v-v^{\prime}\right)[4 u(1-u)]+\delta\left(u-u^{\prime}\right)[4 v(1-v)]\right]\right\},
\end{aligned}
$$

where $S_{+}$and $\Delta_{-}^{[8]}$ are functions of partonic fractions, given in Ref. [ए]]. This result is consistent with the kernel derived in Ref. [14] except for the logarithmic term and the last line. As a special case, if both $\kappa$ and $\kappa^{\prime}$ are color-singlet, our results of $\Gamma_{[Q \bar{Q}(\kappa)] \rightarrow\left[Q \bar{Q}\left(\kappa^{\prime}\right)\right]}$ reproduce the familiar Efremov-Radyushkin-Brodsky-Lepage evolution kernel [며, [20].

\section{Summary}

We have presented an all order QCD factorization formalism for the inclusive production of heavy quarkonia at large $p_{T}$, which provides a systematic approach to study their production at collider energies beyond leading power. Although the rate to produce a heavy quark pair at a smaller distance scale than $1 / m_{Q}$ is suppressed in comparison with the production of a single parton, the probability for the pair to become a heavy quarkonium is larger than that for a single parton to form a heavy quarkonium by fragmenting into a heavy quark pair at a later time. We derived a closed set of evolution equations for both single parton and heavy quark pair fragmentation functions. We pointed out that once we work beyond the leading power, QCD evolution of fragmentation functions with respect to the variation of the factorization scale mixes the heavy quark pair fragmentation functions with single parton fragmentation functions. Such mixing in evolution corresponds to a resummation of the probability for the single fragmenting parton to generate a heavy quark pair from the distance scale of the hard collision, $\sim 1 / p_{T}$, to a scale, $1 / \mu_{0} \sim 1 / m_{Q}$ at which the fragmentation process becomes non-perturbative. We calculated perturbatively the lowest order evolution kernels for all channels of heavy quark pair fragmentation functions, and also derived the first order evolution kernels for a single parton to evolve into a heavy quark pair. As expected 
from the factorization, all calculated evolution kernels are infrared finite. With the perturbatively calculated hard parts in Ref. [[2]], evolution kernels derived here, and model input fragmentation functions in Refs. [15, [6]], we are ready to evaluate the heavy quarkonium production cross section at collider energies.

\section{Acknowledgments}

This work was supported in part by the U. S. Department of Energy under contract Nos. DEAC52-06NA25396 and DE-AC02-98CH10886, and the National Science Foundation under grants No. PHY-0969739 and -1316617.

\section{References}

[1] G. T. Bodwin, E. Braaten, and G. P. Lepage, Phys. Rev. D51, 1125 [Erratum (1995), arXiv:hep-ph/9407339.

[2] M. Butenschoen and B. A. Kniehl, Phys.Rev.Lett. 108, 172002 (2012), arXiv:1201.1872.

[3] K.-T. Chao et al., Phys.Rev.Lett. 108, 242004 (2012), arXiv:1201.2675.

[4] B. Gong, L.-P. Wan, J.-X. Wang, and H.-F. Zhang, Phys.Rev.Lett. 110, 042002 (2013), arXiv:1205.6682.

[5] N. Brambilla et al., Eur.Phys.J. C71, 1534 (2011), arXiv:1010.5827.

[6] G. T. Bodwin et al., (2013), arXiv:1307.7425.

[7] G. C. Nayak, J.-W. Qiu, and G. F. Sterman, Phys. Rev. D72, 114012 (2005), arXiv:hep-ph/0509021.

[8] G. C. Nayak, J.-W. Qiu, and G. F. Sterman, Phys.Rev. D74, 074007 (2006), arXiv:hep-ph/0608066.

[9] Z.-B. Kang, J.-W. Qiu, and G. Sterman, Nucl.Phys.Proc.Suppl. 214, 39 (2011).

[10] Z.-B. Kang, J.-W. Qiu, and G. Sterman, Phys.Rev.Lett. 108, 102002 (2012), arXiv:1109.1520.

[11] Z.-B. Kang, Y.-Q. Ma, J.-W. Qiu, and G. Sterman, (2014), arXiv:1401.0923.

[12] Z.-B. Kang, Y.-Q. Ma, J.-W. Qiu, and G. Sterman, Heavy Quarkonium Production at Collider Energies (II): Partonic Cross Sections and Polarization, in preparation.

[13] S. Fleming, A. K. Leibovich, T. Mehen, and I. Z. Rothstein, Phys.Rev. D86, 094012 (2012), arXiv:1207.2578.

[14] S. Fleming, A. K. Leibovich, T. Mehen, and I. Z. Rothstein, Phys.Rev. D87, 074022 (2013), arXiv:1301.3822.

[15] Y.-Q. Ma, J.-W. Qiu, and H. Zhang, (2013), arXiv:1311.7078.

[16] Y.-Q. Ma, J.-W. Qiu, and H. Zhang, (2014), arXiv:1401.0524.

[17] G. F. Sterman, Phys.Rev. D17, 2773 (1978).

[18] J.-W. Qiu and G. F. Sterman, Nucl.Phys. B353, 137 (1991).

[19] G. P. Lepage and S. J. Brodsky, Phys.Lett. B87, 359 (1979).

[20] A. Efremov and A. Radyushkin, Theor.Math.Phys. 42, 97 (1980). 